\title{
Dynamique verbale et jeux d'identité dans les discours politiques ivoiriens
}

\section{Essé Kotchi Katin Habib}

Assistant à L'université Peleforo Gon Coulibaly (UPGC), Côte d'Ivoire (Costa do Marfim) orcid.org/0000-0001-8356-5936

La force de tout acte d'énonciation est que tout locuteur, lorsqu'il produit un énoncé dans une situation donnée, instaure un certain type de relation: menace, promesse, ordre, etc. avec l'allocutaire. II vise à modifier un état de choses existant, vise à agir sur autrui et le faire agir. Cela passe par l'usage et le positionnement concomitant des mots dits verbes et ceux dits pronoms, ces logos puissants, qui répondent à cette dynamique discursive. L'objectif de cette étude est de montrer comment à partir des verbes épaulés des pronoms, plusieurs identités s'installent et sont installées dans le discours pour créer, in fine, un conglomérat d'univers référentiels.

Mots clés: Verbe. Déclaration et éthique. Discours politique. Lexique et identité.

\section{Dinâmica verbal e jogos de identidades nos discursos políticos da Costa do Marfim}

A força de qualquer ato de enunciação é que qualquer falante, quando apresenta um enunciado numa determinada situação, instaura um certo tipo de relação: ameaça, promessa, ordem etc. com o ouvinte. Visa alterar um estado de coisas existente, visa agir sobre outrem e fazê-lo agir. Isso passa pelo uso e posicionamento concomitante das palavras ditas verbos e aqueles ditos pronomes, este poderoso logos, que responde a esta dinâmica discursiva. 0 objetivo deste estudo é mostrar como, a partir dos verbos de pronomes apoiados, várias identidades se instalam e são instaladas no discurso para criar, in fine, um conglomerado de universos referenciais.

Palavras-chave: Verbo. declaração e ética. Discurso político. Léxico e identidade.

\section{Dinámica verbal y juego de identidad en los discursos políticos marfileños}

La fuerza de todo acto de enunicación es que todo locutor, cuando produce un enunciado en una situación dada, instaura cierto tipo de relación: amenaza, promesa, orden, etc. con el alocutario y apunta a modificar un estado de cosas existentes; a actuar sobre otros y hacerlos actuar. Esto sucede por el uso y el posicionamiento concomitante de verbos y pronombres, estos logos poderosos, que responden a esta dinámica discursiva. El objetivo de este estudio es mostrar cómo a partir de los verbos apoyados en los pronombres muchas identidades se instalan y son instaladas en el discurso para crear, en fin, un conglomerado de universos referenciales.

Palabras clave: Verbo. Declaración y ética. Discurso político. Léxico e identidad.

\section{Verbal dynamics and identity games in Ivorian political discourse}

The strength of any act of enunciation is that any speaker, when producing a statement in a given situation, introduces a certain type of relationship - threat, promise, order, etc. - with the allocutary. It aims to change an existing state of affairs, to act on others and to make them act. This involves the concomitant use and positioning of verbs and pronouns, these powerful logos that responds to this discursive dynamic. The objective of this study is to show how, from the verbs supported by pronouns, several identities are conveyed and are conveyed in discourse to create, in fine, a conglomerate of reference universes.

Keywords: Verb. Declaration and ethics. Political discourse. Lexicon and identity. 


\section{Introduction}

La présente réflexion porte sur le jeu des verbes épaulés par les déictiques personnels et l'implication identitaire que draine leur énonciation. En grammaire, le verbe est cet élément exprimant un procès, un état ou un devenir, variant, dans de nombreuses langues, en nombre, en personne et en temps et ayant pour fonction syntaxique de structurer les termes constitutifs de l'énoncé. II est le mot qui donne une coloration sémantique à l'idée énoncée. P. Monneret et R. Rioul (2009, p. 87) disent qu'il est la pièce syntaxique "révélatrice de l'architecture d'ensemble" du discours. Dans l'énoncé: "J'engage toutes les forces de défense et de sécurité", c'est la lexie "engager", sous l'impulsion du “j'”, qui, comme un indicateur de mouvement, donne le branle au discours et incite, dans le même temps, le récepteur à l'action souhaitée: "invitation à participer à'. C'est un aspirateur lexical qui tire tout le discours vers son accomplissement scénique et sociolinguistique. Ce dynamisme verbal est florès chez les locuteurs politiques Ivoiriens, et même chez les locuteurs politiques tous azimuts. Le verbe participe, en plus de l'action, à la création d'une identité sociocognitive et plante dans le discours un infini conflit de positionnement. Quelles sont les catégories qu'on y rencontre et comment fonctionnent-elles? Quels sont, à partir dudit élément, les identités socio-discursives qui se déploient de par la langue? L'analyse du contenu, comme méthode, nous prêtera ses outils pour conduire cette réflexion.

\section{Définitions des notions, méthodologie et corpus}

Ce cadre élucide les concepts de dynamique verbale, éthos et jeux d'identités, pose la méthode mobilisée pour circonscrire l'objet d'étude et situe le corpus.

\subsection{Définition des notions}

\subsubsection{Dynamique verbale}

Du point de vue de la linguistique, de la grammaire et même des théories de l'énonciation, le verbe (du latin verbum: mot, verbe) constitue avec le nom la catégorie de mots ou partie du discours principale de toute langue. La grammaire traditionnelle présente le verbe en opposition au nom grâce à sa capacité d'exprimer un procès, en traduisant une action accomplie (il lit un livre) ou subie par le sujet (il a été déçu par ce livre), ou un état (il est heureux d'avoir lu) ou des modifications du sujet (il ne veut plus de ce livre). À cet aspect sémantique la linguistique contemporaine, de tendance majoritairement distributionnaliste et structuraliste, oppose une approche 
morphosyntaxique, rejetant toute définition du verbe fondée sur son rapport avec un aspect de la réalité qui le distinguerait du nom (fonction référentielle) pour privilégier sa fonction de mise en relation des autres composants de la phrase. Or, cette fonction référentielle historiquement dévolue au nom est bien applicable au verbe. Son énonciation dans certains cas, comme dans les discours politiques (ivoiriens), est fortement orientée vers un univers référentiel et comme le soutiennent J. Dubois, M. Giacomo, L. Guespin, C. Marcellesi, J.-B. Marcellesi et J.-P. Mével (2018, p. 506) “dans une formulation lexico-sémantique des énoncés, le verbe (ou prédicat) est la fonction essentielle qui gouverne les arguments". Gouverner les arguments c'est ce que R. Ghiglione, M. Bromberg, E. Friemel, C. Kekenbosch et J.-C. Verstiggel (1990) ont nommé un "programme cognitivo-discursif" qui vise, selon eux, "à mettre en scène un "monde" possible ou réel". Ce qui suppose, à minima, une correspondance entre activité cognitive et concrétisation langagière: il y a dynamisme. Ces différents postulats s'appliquent naturellement au verbe, élément déterminant de la proposition, au sens que lui donne, notamment, É. Benveniste (1970, p. 154) "la fonction verbale est double: fonction cohésive, qui est d'organiser en une structure complète les éléments de l'énoncé; fonction assertive consistant à doter l'énoncé d'un prédicat de réalité". Dès lors, l'univers lexical du verbe est l'ensemble de tous les verbes autour desquels pivotent les idées forces dans les discours des politiques Ivoiriens (et de Laurent Gbagbo en particulier, puisque c'est sur ses discours que nous portons le regard). R. Ghiglione parle de "référents noyaux". Autrement dit, les mots-gouverneurs autour desquels s'ordonnent les réalisations langagières. Dans l'environnement discursif politique, le langage n'est pas seulement un simple moyen d'information mais il est aussi, et surtout, une puissante machine d'action, d'exercice de pouvoir, d'autorité et d'influence, selon les mots de $P$. Charaudeau (2005), et de création d'identité. Or cette création qui se fait à partir de la synergie de l'élément verbe épaulé par le pronom personnel, stratifie le corps sociopolitique et dorénavant il est le reflet d'un ensemble de caractères propres à un individu ou à un groupe: il y a donc ethos et jeux d'identités.

\subsubsection{Ethos et jeux d'identités}

II faut distinguer, avec M. Stasilo (2012, p. 68), l'èthos - du grec ancien êthos, pluriel ếthê, où "è" est la transcription de la lettre grecque êta - et l'éthos - du grec ancien éthos, où "é" est la transcription d'epsilon. Le premier mot est associé au caractère, au comportement physique, à l'état d'âme. Cette catégorie est souvent considérée du point de vue moral. Le mot "èthos est souvent traité dans la rhétorique qui se focalise sur l'art de persuader. Il est fortement lié à la construction de l'image que le locuteur donne à la fois de lui-même et "de ceux auxquels il s'adresse, de leur affectivité" (DUBOIS et al., 2018, p. 187) dans son activité discursive. Le sens de l'ethos est allé de glissando en glissando au fil des siècles. En effet, dans ses glissements historiques comme dans les 
ré-exploitations qui en sont faites aujourd'hui, la notion d'ethos, toute simple qu'elle puisse paraître au premier abord, pose de multiples problèmes si l'on veut la cerner avec quelque précision. Déjà, dans la micro-sociologie de Goffman, nous retenons que l'ethos est la présentation de soi. II pose que dans les échanges de la vie quotidienne, les individus se présentent d'une certaine manière selon la finalité de l'échange. Il "élargit le champ de l'ethos en appliquant la construction d'une image de soi à tous les échanges de la vie quotidienne" (KOFFI-LEZOU, 2012, p. 5). Lesquels échanges sont bâtis sur des référents noyaux, des mots-verbes, qui gouvernent sémantiquement l'énoncé et engagent à la fois la cognition et la personnalité même du locuteur et de l'allocutaire. De ce fait, le constat, dans la perspective de Koffi-Lézou (2012), est que le locuteur politique Ivoirien, par l'emploi qu'il fait d'un verbe donne une coloration particulière à son discours, dans lequel il s'investit, au point où aucun mot n'est énoncé ex-nihilo. Les identités se signalent et se construisent alors dans le discours à tour de rôle et selon les objectifs de la communication: il y a jeu d'identité. La distribution de rôles repose sur la subtilité dans l'emploi du verbe (et de certaines pièces adductrices) qui phagocyte la distanciation entre le locuteur et son adversaire socio-politique avant d'établir un rapprochement à valeur comparative. L'étude de cet élément linguistico-discursif passe alors par une méthodologie que nous déclinons comme suit.

\subsection{Méthodologie}

Nous utiliserons l'analyse lexicale (dorénavant $A L$ ), qui contient intrinsèquement l'analyse du contenu et l'analyse statistique, pour éclairer les différents aspects du langage susceptibles de fournir des indices sur la pensée, les représentations du locuteur. L'analyse des adaptations personnelles que l'énonciateur fait subir, consciemment ou non au mot. C'est une analyse des modalités du dire qui se concentre sur les mots plutôt que sur le texte dans son ensemble. M.-L. Gavard-Perret et J. Moscarola (1998) dans "Enoncé et ou énonciation? deux objets différents dans l'analyse lexicale en marketing" qui y ont proposé une relecture même de l'AL, distinguent l'énoncé de l'énonciation en passant en revue différentes techniques et en précisant les deux fonctions possibles de l'AL dont l'analyse descriptive ou l'approximation des contenus, d'une part, et l'analyse interprétative ou la statistique des actes du langage, d'autre part. L'AL permet donc de décrire et d'interpréter le dit (l'énoncé) sur la base d'indices statistiques qui présentent les fréquences d'emploi. À partir de ce moment, elle s'intègre à la démarche originelle d'analyse du contenu au sens de Berelson (1954), cité par M.-L Bavard-Perret et J. Moscarola (1998), qui pose qu'il s'agit d'une

[...] analyse systématique, objective, quantitative, qui se doit de ne pas être un simple commentaire, et doit aboutir à une production de données quantitatives [...] L'idée de base de l'analyse lexicale est simple: substituer à l'étude d'un texte celle de l'ensemble des mots qui le composent. Tout corpus étant composé d'éléments 
répétés, l'examen de son lexique (liste des mots différents contenus dans le corpus) présente l'avantage de réduire le volume et la complexité du matériau à analyse ${ }^{1}$

L'AL, sous cet angle, touche le contenu du texte et dresse, à l'aide d'outils de la statistiques lexicale ou la linguistique quantitative, la fréquence (le nombre d'occurrence) de l'unité à étudier dans le corpus; car:

[...] étudier le sens et/ou la distribution d'une unité ne suffit pas, il faut aussi tenir compte de sa fréquence. Là, nous somme dans le champ dévolu à la lexicométrie qui est une méthodologie d'étude du discours, qui se veut exhaustive, systématique et automatisée (CHARAUDEAU; MAINGUENEAU, 2002, p. 342-343).

Elle vise à mesurer la quantité des mots d'un texte, plus précisément les lexèmes qui couvrent toutes les classes: substantifs, pronoms, adjectifs, temps et mode d'un verbe, etc. La fréquence qui calculera les occurrences diverses devient un attribut positif et concret dans l'étude à mener. C. Desmarais et J. Moscarola (2004) soutiennent qu'en "tirant parti de la redondance de la langue pour examiner les termes les plus fréquemment employés, on se fait beaucoup plus rapidement une idée". Ils poursuivent en disant qu'examiner alors les substantifs, les verbes, les adjectifs les plus fréquents permet une approche très efficace du corpus et donne une idée de son contenu (l'énoncé), mais met à jour les traits caractéristiques de l'énonciation.

\subsection{Corpus}

La notion de corpus peut être définie comme étant un ensemble d'énoncés représentant un échantillon de la langue. J. Sinclair (1991, p. 2) la définit comme étant: "un ensemble de morceaux de langue qui sont sélectionnés et classés en fonction de critères linguistiques explicites afin d'être utilisé comme un échantillon de la langue". Comme on le voit, le corpus est une réalité métonymique d'un grand ensemble d'éléments considérés pour leur caractère spécial. Partant, le support de cette étude, est un morceau de la vaste surface de production langagière de Laurent Gbagbo; exprésident de la République de Côte d'Ivoire (de 2000 à 2010). C'est une banque de données constituée de trente discours officiels recueillis sur le site de la présidence de la République de Côte d'Ivoire ${ }^{2}$. C'est dans un contexte général de crise que ce corpus a été énoncé. Le discours étant l'association d'un texte et de son contexte, nous soutenons qu'il s'agit d'un corpus spécialisé de crise car tous les sujets qui y sont abordés font référence à la crise tous azimuts que traverse la Côte d'Ivoire. À la vérité, la crise ivoirienne de 2000 à 2010 a impulsé une pratique linguistique inhabituelle. Cela à infusé dans le parler ivoirien un caractère spécial au point où tous les mots et autres

\footnotetext{
${ }^{1}$ Consulté le 1er septembre 2016 sur http://www.e-marketing.fr/Definitions-Glossaire/Analyse-lexicale240497.htm\#d0otl83r4ORrFewX.99

$2 \mathrm{http}: / / \mathrm{www}$.presidence.ci
} 
expressions étaient des charges connotatives à entrées multiples. Cette banque de données est représentative des dix années de pouvoir de l'énonciateur car elle reprend de façon générale le récit des moments difficiles. Le principal critère relevant du choix de ces discours réside dans une perspective diachronique qui offre la possibilité d'observer maintes variables dans l'évolution de la situation de crise. Lesquelles variables densifient l'univers linguistique donnant ainsi matière à analyse. Ici, nous portons le regard sur le jeu du verbe épaulé par les déictiques personnels.

\section{Univers référentiel du verbe: de l'osmose d'identités}

Nous travaillons avec le logiciel TROPES ${ }^{3}$. Avant de commencer les analyses, le logiciel a découpé le corpus en partie et a compté le nombre de chaque verbe en donnant sa fréquence. Nous présentons les deux scénarios (voir Annexes).

La cartographie de la surface verbale du corpus présente ce qui précède. De l'occurrence la plus élevée (2660) à la plus faible (0003), le verbe désigne un univers référentiel dense et composite à qui est adressé le discours. Son usage partage la société ivoirienne de la crise en groupes d'actants qui sont eux-mêmes cachés dans les embrayeurs (pronoms) qui aident le verbe à opérer un positionnement et une détermination. Nous les avons catégorisés de façon utile pour nos objectifs. Selon R. Ghiglione et al. (1985, p. 40) "les catégories verbales sont ici réduites à trois classes: statifs, factifs et déclaratifs". Soit la répartition suivante:

Tableau 1 - Catégories verbales dans le corpus

\begin{tabular}{|l|l|l|}
\hline Types de verbes & Occurrences & Taux (en \%) \\
\hline Factif & 6214 & 43,9 \\
\hline Statif & 4717 & 33,3 \\
\hline Déclaratif & 3045 & 21,5 \\
\hline Performatif & 175 & 1,2 \\
\hline TOTAL & 14149 & 99,9 \\
\hline
\end{tabular}

On appellera verbe factif tout item défini lexicalement comme renvoyant à la transcription langagière d'une action et verbe statif tout item renvoyant à la transcription langagière d'un état ou d'une possession. Etre ou avoir n'étant pas considérés comme auxiliaires. Les déclaratifs, tout verbe défini lexicalement comme renvoyant à une déclaration sur un état, une action, un être, un objet. Toutefois, cette classification ne peut être considérée comme satisfaisante pour nous c'est pourquoi nous avons adjoint

\footnotetext{
3 TROPES, Logiciel développé par Pierre Molette et Agnès Landre sur la base des travaux de Rodolphe Ghiglione, version 8.4.4 (buid 0004), http://www.tropes.fr
} 
la catégorie des performatifs, même si elle est quelque peu inspirée des travaux de classification des verbes menés depuis une trentaine d'années. On la considérera comme la transcription langagière d'un acte par le langage et dans le langage. Elle ne sera pas étudiée ici. L'univers référentiel du verbe contient une multitude d'identités qui tantôt se mêlent pour devenir une seule et même image tantôt s'entretiennent dans une relation dysphorique et de conflit, de rejet et de différenciation: il y a donc osmose.

\subsection{Les verbes factifs ou l'identité d'action}

L'enjeu du discours politique est la recherche du consentement d'une certaine collectivité sociale. C'est un "despote puissant" (DORNA, 1995, p. 132) et un instrument d'action qui produit un lien d'interaction entre les membres de ladite collectivité. Y être c'est participer aux interactions que déploient la langue qui offre plusieurs chemins à la pensée subjective pour devenir extérieure et commune. Autrement, c'est par le vocabulaire, la grammaire, la syntaxe et les sons que la langue permet à l'individu d'extérioriser, partager son for intérieur. La quintessence des actions ainsi libellées dans ses propos réside dans la valeur sémique des verbes employés en situation de discours. Ici, les factifs sont des verbes qui expriment essentiellement une ou des actions posées par l'énonciateur. Mobilisant 6124 occurrences sur 14149 soit $43,9 \%$ de la surface verbale du corpus, cette prédominance s'explique par le fait que l'énonciateur s'attarde sur les actions menées, les actions qu'il mène ou qu'il va mener. Cela dépendant des enjeux et lieux de l'énonciation. Chaque verbe énoncé est le versant d'une action spécifique. L'action en question peut avoir été menée par un autre individu désigné ou non dans l'espace discursif. L'emploi d'un verbe factif cache et dévoile à la fois une identité socio-politique qui peut refléter des relations de paix ou de conflit. Certains verbes se retrouvent à caractériser à la fois l'énonciateur et ses partisans et l'adversaire politique. Soient les extraits suivants:

Je m'apprêtais à continuer mon travail quand j'ai été informé de graves évènements qui se dérouleraient [...] Ce qui s'est passé, et qui continue de se passer ne nous y trompons pas, c'est une tentative de coup-d'état [...] Nous avons réussi à neutraliser tous ceux qui s'étaient infiltrés à Abidjan. Et Nous allons continuer le travail en poursuivant systématiquement tous ceux qui se sont retranchés dans les quartiers précaires. Nous allons les nettoyer! Enfin, Nous allons continuer notre progression vers le Nord [...] On nous impose une bataille, menons la avec courage (D5, 20.09.02) / / Un grand drame se produisait en Afrique [...] (D7, 22.11.02) / Vous m'avez accompagné à Paris dans une escorte émouvante [...] Je ferai tout ce que je peux pour mériter toujours votre confiance $(\underline{D 9}, . .02 .03)$ / Vous m'avez fait confiance en me mettant à la tête de ce pays / Tout ce que j'ai commencé, je le continuerai / Les gens ont continué à gouverner dans les années 1970,1980, 1990, 
comme si on était dans les années 1960 / ils ont gouverné tous et moi j'étais dans l'opposition (D4, ..11.01) ${ }^{4}$

Ces extraits fonctionnent avec pour noyaux verbaux: ferai; avez accompagné; avez fait; mettant; impose; menons; chercher; produisait; reprenne; aillent; jouer; permettre; est intervenu; former; avons réussi; allons continuer; poursuivant; sont retranchés; allons nettoyer; apprêtais; dérouleraient; s'est passé; ai commencé; gouverner.

Tous ces verbes indiquent des actions bien déterminées que l'énonciateur présente à l'auditoire comme un récit. À chacune des actions énoncées est affiliée un sujet-causateur qui devient ainsi le porteur de la valeur connotative du verbe énoncé. Ce sont des verbes causatifs car ils deviennent les déclencheurs de présuppositions et de représentation de l'individu ou du groupe d'individus responsable de l'action dénotée par l'infinitif. Selon la temporalité, l'action peut être rétrospective, instantanée ou prospective en contexte et en situation d'énonciation. Le discours est ainsi dans un perpétuel passéprésent-futur. Selon le contexte d'énonciation, le jeu des pronoms et des syntagmes nominaux qui appuient l'intensité des verbes employés, trois identités causatives se dégagent de l'extrait: les partisans, les adversatifs et les modérées.

\subsubsection{Verbes factifs: de l'identité de partisan}

Les verbes factifs partisans sont ceux qui désignent les actions menées par l'énonciateur et/ou le peuple représentés soit par le je soit par le nous inclusif ou le nous exclusif. C'est par le positionnement de ces embrayeurs de personne que le verbe prend toute sa valeur référentielle. Dans les énoncés

Je ferai tout ce que je peux pour mériter toujours votre confiance / Vous m'avez accompagné à Paris dans une escorte émouvante / Vous m'avez fait confiance / Je m'apprêtais à continuer mon travail / Tout ce que j'ai commencé, je le continuerai.

Les actions prospectives de "faire" et de "continuer" et les rétrospectives "apprêter" et "commencer" ont pour sujet actantiel je qui indique la présence de l'énonciateur dans le champ discursif tandis que dans la rétrospective l'action d"'accompagner" et de "faire" sont déployées pour le peuple-collaborateur désigné par vous. Par ces verbes l'énonciateur ne déroge pas à son intention de préserver son image et rendre au peuple la reconnaissance qui lui est due. C'est à dessein que l'action de "faire" est suivie du substantif "confiance" et la fascination verbale logée en arrière-plan de l'action

\footnotetext{
${ }^{4}$ En vue d'éviter d'alourdir indéfiniment notre texte par des notes de bas de page, nous avons jugés d'attribué des codes contenant en initial la lettre " $D$ " suivi du numéro du discours, le tout suivi de la date référentielle à laquelle le discours a été prononcé. Exemple " D1, 24.10.00 " pour indiquer qu'il s'agit d'un extrait du discours 1 prononcé le 24 octobre 2000.
} 
d'"accompagner" est révélée dans l'adjectif "émouvante". Ces verbes sont révélateurs de la participation du peuple au déroulement des actions dans les interactions sociales. Le locuteur incite le peuple partisan à maintenir le cap des actions entreprises et joue surtout sur la fibre patriotique en utilisant un embrayeur englobant. Dans l'énoncé:

Nous avons réussi à neutraliser tous ceux qui s'étaient infiltrés à Abidjan. Et Nous allons continuer le travail en poursuivant systématiquement tous ceux qui se sont retranchés dans les quartiers précaires. Nous allons les nettoyer! Enfin, Nous allons continuer notre progression vers le Nord (D5, 20.09.02)

Les actions de "réussir" "atteindre son objectif", "continuer" "poursuivre la même action", "nettoyer" "tuer les assaillants, les éliminer, les mettre hors d'état de nuire", en contexte connoté, ont pour sujet le nous-inclusif. Par cet embrayeur le je et le vous conjuguent leurs forces pour agir. Statistiquement élevé avec plus de 3000 occurrences, les items factifs partisans poursuivent un seul projet: mettre le peuple au cœur des évènements. D'où le choix de ces verbes.

Les verbes d'action ont troqués leurs sens premiers pour des sens nouveaux, valables dans le contexte de la crise ivoirienne. Je, vous et nous sont les indices référentiels et identitaires de l'énonciateur, du partisan et du peuple. C'est l'ensemble des verbes avec lesquels ils sont en interaction dans le corpus qui constitue l'univers lexico-référentiel des verbes factifs partisans. Au nombre de 119 entités verbales, chacune apparait dans son aspect et dans son action spécifique. En filigrane, elles poursuivent le projet de traduire les lignes d'action de l'énonciateur et poser les différentes identités actantielles sur la scène: politique, répressive et constructive. La Figure 1 reprend la distribution selon ces catégories actantielles énumérées.

Par action d'opposition, il faut entendre toute action physique, toute action de force menée en réponse à une action de l'adversaire socio-politique. Les actions politiques, quant à elles, sont le pendant d'une lutte idéologique qui dévoile la vision et l'organisation du pouvoir de l'énonciateur. Ces verbes sont portés sur des appréhensions personnelles qui prennent, avec les embrayeurs du peuple (en l'occurrence le nous-inclusif), une coloration collective. Le but est de faire participer et de faire se sentir la collectivité dans ces actions. C'est en cela qu'au cœur des actions constructives se retrouvent des idées d'union autour de l'intérêt général de la collectivité. À côté de ses actions marquées par les factifs partisans, un autre type d'action s'offre à analyse: les actions adversatives. 
Figure 1 - Univers lexico-référentiel des verbes factifs partisans

\begin{tabular}{|c|c|c|}
\hline & Factifs Partisans & \\
\hline $\begin{array}{l}\text { Action d'opposition } \\
\text { Affronter, arrêter, } \\
\text { assumer, battre, briser, } \\
\text { céder, débarrasser, } \\
\text { défendre, déférer, } \\
\text { descendre, dresser, } \\
\text { efforcer, empêcher, } \\
\text { équiper, exercer, heurter, } \\
\text { importuner, jeter, lutter, } \\
\text { ordonner, soulever, } \\
\text { tomber }\end{array}$ & $\begin{array}{l}\text { Action politique } \\
\text { Accorder, asseoir, conférer, } \\
\text { consacrer, consigner, } \\
\text { contourner, déléguer, } \\
\text { démêler, diriger, élire, faire, } \\
\text { gouverner, incarner, instituer, } \\
\text { inviter, marcher, mener, } \\
\text { organiser, parcourir, pousser, } \\
\text { préparer, présider, prêter, } \\
\text { préserver, rechercher, } \\
\text { réécrire, reprendre, servir, } \\
\text { sortir, soumettre, tendre, } \\
\text { terminer, tracer, tranquilliser, } \\
\text { verser }\end{array}$ & $\begin{array}{l}\text { Action constructive } \\
\text { Accompagner, affecter, } \\
\text { apporter, baptiser, bâtir, } \\
\text { commémorer, commencer, } \\
\text { consolider, continuer, courir, } \\
\text { dérouler, édifier, effectuer, } \\
\text { élaborer, embrasser, emmener, } \\
\text { entendre, essayer, établir, } \\
\text { examiner, fâcher, faillir, finir, } \\
\text { fonder, former, fraterniser, } \\
\text { garder, habituer, honorer, } \\
\text { incliner, instaurer, laisser, } \\
\text { léguer, lier, mettre, montrer, } \\
\text { obtenir, pardonner, passer, } \\
\text { précipiter, prendre, rassembler, } \\
\text { réunir, saluer, subir, toucher, } \\
\text { tranquilliser, transférer, } \\
\text { transmettre, traverser, unir, } \\
\text { veiller. }\end{array}$ \\
\hline
\end{tabular}

\subsubsection{Verbes factifs: de l'identité adversative}

Les verbes factifs adversatifs, contrairement à la première classe, indiquent les actions des adversaires socio-politiques. Les verbes sus-dégagés peuvent apparaitre ici aussi, mais avec un positionnement différent, le mot n'ayant de sens qu'en contexte. Les embrayeurs vous, ils, on, eux désignant l'adversaire en contexte, sont les indicateurs identitaires de la valeur référentielle des verbes employés. Ce sont eux qui épaulent le verbe dans sa significativité et dans son efficience à caractériser et porter l'identité de l'adversité et par ricochet ses actions.

On nous impose une bataille, menons la avec courage / Les gens ont continué à gouverner dans les années 1970,1980, 1990, comme si on était dans les années 1960 / ils ont gouverné tous et moi j'étais dans l'opposition.

Les verbes "imposer" du latin imponere pris, ici, dans le sens de "déclarer, chercher querelle, inviter à"; "continuer", du latin continuare pris, ici, dans le sens de "demeurer, rester dans une logique" et "gouverner" du latin gubernare, pris dans le sens de "commander, gérer les affaires du pays", sont dits verbes factifs adversatifs en ce qu'ils 
désignent des actions qui sont coptées par les nommeurs identitaires "on", "ils" et "gens" représentant l'adversaire dans l'explicite du discours.

La contrainte qui se dégage de cette action est renforcée par le nom "bataille" qui trouve une réponse répressive dans le substantif "courage" qui positionne le partisan à travers le "nous" et le verbe "mener" à la même personne. D'une près de 3000 occurrences, l'énonciateur, par ces verbes, déploie un univers d'actions niées et reniées à l'actif de ses adversaires. A priori, c'est une ligne de dérèglement, de modification d'un ordre socio-politique. Par ces verbes, l'énonciateur présente l'adversaire comme une source de déséquilibre, de violence et dévoile en même temps deux types identitaires d'actants: I'homme de force et le politique. Deux groupes essentiels d'actants sont caractérisés à partir de la distribution verbale dans la Figure 2:

Figure 2 - Univers lexico-référentiel des verbes factifs adversatifs

\begin{tabular}{|c|c|}
$\begin{array}{c}\text { Alimenter, armer, atteindre, battre, boucher, } \\
\text { bouleverser, commettre, compromettre, } \\
\text { débusquer, désarmer, égorger, emparer, } \\
\text { empêcher, encercler, foncer, frapper, fusiller } \\
\text { imposer, mitrailler, munir, nuire, préparer, } \\
\text { provoquer, recruter, renverser, réussir, } \\
\text { tenter, tirer, tomber, trébucher }\end{array}$ & $\begin{array}{c}\text { Accepter, accompagner, circuler, } \\
\text { commencer, continuer, créer, diriger, } \\
\text { diviser, écarter, évertuer, faire, } \\
\text { mesurer, mettre, obtempérer, partir, } \\
\text { passer, percevoir, plaindre, pomper, } \\
\text { reconnaitre, réhabiliter, réjouir, rentrer, } \\
\text { retourner, sauter, tromper }\end{array}$ \\
\hline
\end{tabular}

Certains verbes se retrouvent tant dans la classe partisane qu'adverse. Cela s'explique par le fait que dans la démolition de l'image de l'adversaire, l'énonciateur procède par des parallélisme à forte valeur de comparaisons dans la manière d'exécuter, de mener ou de réaliser l'action dénotée par le verbe. En témoigne cet énoncé:

Pendant que nous préparions les conditions de la prospérité et de la démocratie, ils préparaient la guerre $(\underline{D} 8,30.12 .02)$

Le verbe "préparer" du latin praeparare de prae "pré-" et parare "parer" qui stipule "se mettre en état de faire quelque chose", ici, est le reflet de deux actions distinctes. La première occurrence désigne l'action de l'énonciateur et son groupe partisan représentés dans le "nous" opposé à "ils" en antéposition dans la deuxième occurrence. Le verbe, dès lors, exprime une positivité dans l'action du peuple et une négativité dans l'action de l'adversaire. Ce dualisme est appuyé dans l'opposition des substantifs 
"prospérité", "démocratie" versus "guerre". II en est ainsi de plusieurs autres verbes qui ont le même comportement discursif. Le contexte d'énonciation de ces verbes est marqué par des évènements qui dénaturent, salissent et entachent de méfiance continuelle, la qualité de vie dans la société ivoirienne.

Par action de force, il faut entendre toute action de rudoiement menée par l'adversaire sur le peuple Ivoirien. Les verbes utilisés pour ce groupe d'adversaire laissent transparaitre une violence et une rupture de l'équilibre dans la construction d'une communauté de destin. Ils sont, en effet, la vitrine identitaire de tous les actes perpétrés contre l'énonciateur et le peuple Ivoirien. L'action politique adverse évolue pour la plupart du temps en comparaison avec ses propres actions. La disqualification des actions politiques de l'adversaire ressort essentiellement dans les verbes "intoxiquer" et "pomper" dans l'extrait:

II est bon que devant la presse internationale, on dise tout parce que les gens intoxiquent. Et comme ils sont en Europe, et que les européens ne connaissent pas nos réalités, on les pompe et ils croient naïvement $(\underline{\mathrm{D} 10, . .03 .03})$

Ces deux verbes "intoxiquer" du latin intoxicare de in- (élément négatif du latin) et toxicum (toxique) qui signifie "empoisonner" et "pomper" qui signifie (déplacer, élever un fluide à l'aide d'une pompe, en l'attirant). Faire un mouvement (alternatif, rotatif) comme pour pomper selon Le Grand Robert. En français d'Afrique signifie: Gonfler quelque chose, un pneu, etc. avec une pompe; en ivoirais ou simplement français ivoirien: "berner quelqu'un, mentir à quelqu'un, tromper quelqu'un à propos de quelque chose, gonfler l'esprit de quelqu'un par des mensonges" sont révélateurs de l'opinion que se fait l'énonciateur des actions politiques adverses. La propagande, puisque c'est le sens du verbe "intoxiquer" dans le contexte de la crise ivoirienne, le bourrage de cerveau et le masquage des réalités, ici vécues, sont, pour lui, la seule arme que l'adversaire politique détient. Toujours sans le nommer, il positionne l'adversaire comme la source de déséquilibre et de perdition du peuple Ivoirien.

La crise ivoirienne en discours est le croisement de plusieurs actions émanant de plusieurs identités actantielles: l'énonciateur et le peuple, l'adversaire socio-politique et les actants dits "extérieurs-au-peuple" qui constituent le troisième angle dans ce triangle énonciatif.

\subsubsection{Verbes factifs: de l'identité de l'actant-extérieur}

Les verbes factifs modérés sont des verbes qui expriment dans les discours de Laurent Gbagbo les actions de personne ou groupe de personnes extérieures qui interviennent dans la crise ivoirienne. Cet extérieur, pour rappel, n'est ni adversaire, ni partisan d'où l'aspect de la modération. Apparaissant en près de 2000 occurrences, ses 
actions sont menées en vue de ramener l'énonciateur-peuple et les adversaires à un consensus, à un accord en vue d'éradiquer le mal de la crise militaro-politique. C'est la neutralité de ces sujets dits extérieurs qui confère aux verbes ainsi utilisés la valeur d'actions modérées. Soit l'extrait suivant:

La CEDEAO doit former un groupe de contact pour s'interposer [...] J'ai demandé donc à la France de jouer, pour ce moment, pendant une semaine, le rôle de force tampon (6, ..10.02) / Le secrétaire Général de I'ONU, Koffi ANNAN qui a suivi les progrès accomplis par la Côte d'Ivoire sur la voie de la réconciliation (D8, 30.12.02) / Le GTI est venu et a parlé. Je ne vais pas lui répondre (D15, ..09.06)

Les verbes "former", "jouer", "suivre", "venir" sont des actions neutres dans l'espace discursif de la crise car elles sont menées par les sujets "CEDEAO", "France", "Koffi Annan", "GTP" qui sont des instances extérieures à l'énonciateur-peuple et au groupe d'adversaire. Ces verbes, contrairement aux deux autres groupes gardent, un tant soit peu, leur valeur sémique de base. Ces actions visent à maintenir une certaine stabilité de la situation de crise comme on peut le voir en fin de chaque énoncé dans les indices subséquents: "interposer", "force tampon", "réconciliation". Tous les groupes actantiels évoluent dans un conglomérat d'actions qui prennent à chaque apparition une forme et une identité nouvelle. Ici, l'énonciateur montre toutes les actions entreprises par l'actant-extérieur à partir de deux lignes directrices: restauratrices et opaques.

Figure 3 - Univers lexico-référentiel des verbes factifs extérieurs

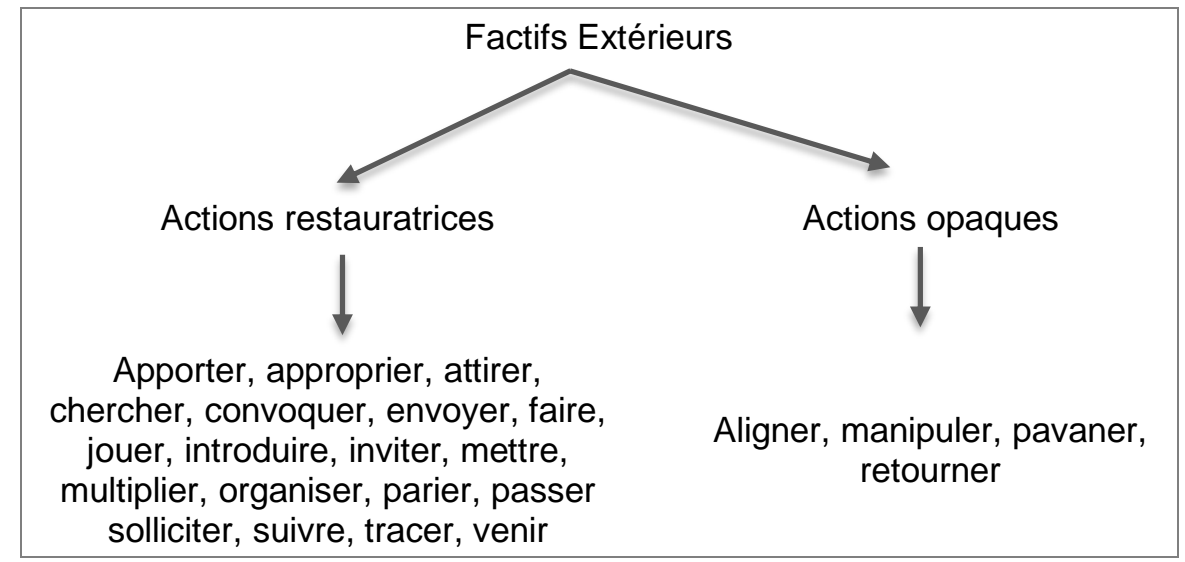

Bien qu'il soit neutre, ce groupe n'est pas si homogène dans le positionnement qu'en fait l'énonciateur. II y a, d'un côté, un groupe favorable dont les agissements visent surtout à rétablir l'autorité et le pouvoir d'État et, inversement, certains verbes sont témoins d'une aversion qu'il porte à une autre frange qui agit de façon opaque. C'est, par exemple, le cas du dernier extrait sus-indiqué: "Le GTI est venu et a parlé. Je ne vais pas lui répondre". Le refus de donner réponse à l'action du GTI cache une attitude d'éloignement, de répulsion vis-à-vis des agissements de ce groupe pour qui, d'ailleurs, 
il allègue les verbes "manipuler, pavaner, retourner, aligner" qui ont des connotations fortement péjoratives. Par-là, il montre que l'actant-extérieur n'a toujours pas été si neutre dans le déroulement des actions caractéristiques de la crise.

Tous autant qu'ils sont, les postes pourvus dans le schéma actantiel de la crise ivoirienne révèlent des actants aux visages disparates. Chacun à son niveau pose des actions mais aussi exprime ses états et ses intérêts. Le discours est ainsi parsemé de tous ces éléments qui montrent leurs positions, leurs états et leurs univers diversiformes. Autrement dit, il met "en scène des acteurs, des actes et différents descripteurs spécifiant ces acteurs et ces actes" (GHIGLIONE et al., 1985, p. 81). Derrière l'énonciation d'une action menée se cache un état, une certaine notion de possession que l'actant énonciatif veut communiquer. Et cela passe par le verbe, contenant de ce contenu énonciatif. L'étude des verbes factifs a permis de ressortir des traces lexicales d'actions à portée politique, sociale, répressive, répulsive et restauratrice. Voyons ce qu'il en est des verbes statifs.

\subsection{Les verbes statifs}

Avec J. Dubois et R. Lagane (2005, p. 18), disons que "la phrase est l'élément fondamental du discours, constitué d'une combinaison de groupe de mots, elle est douée de sens. On distingue les mots selon leur sens, leur forme et leur fonction". La fonction de certains mots est de référer à des états comme dans cet énoncé: "Mon pays est attaqué!" ou désigner des propriétés. Ce rôle est pour la plupart du temps jouer par des verbes communément appelés "verbes d'état". Pour cette étude, ils seront repris sous l'appellation de verbes statifs.

Dans les discours de Laurent Gbagbo, ces verbes tournent autour des archilexèmes "être" et "avoir". Les verbes statifs apparaissent en 4717 occurrences dont 2636 occurrences pour le verbe "être" dévoilé dans toutes les déclinaisons personnelles et temporelles, 1034 occurrences pour le verbe "avoir" paraissant aussi dans toutes les tournures personnelles et temporelles et 1047 occurrences pour tous les autres verbes statifs du corpus, appelons-les verbes statifs-vicaires. Ces prépondérances peuvent s'expliquer par le fait que l'énonciateur par son je-vous-nous exprime essentiellement des états et se sert beaucoup de ces deux formes pour renforcer l'intensité expressive d'autres verbes statifs. "Être" et "avoir" dans ces conditions sont, certes, des verbes auxiliaires mais des verbes statifs auxiliaires. Dans l'action du discours, les verbes statifs sont réputés dans la mise en relation du sujet et son attribut. Soit l'extrait suivant:

J'ai organisé le forum pour la réconciliation nationale. A cette occasion, tous ceux qui avaient quelque chose à dire ont parlé [...] nous avons dit non! [...] Nous avons fait en sorte que tous ceux qui étaient en exil, rentrent en Côte d'Ivoire et que tous 
ceux qui étaient en exil à l'intérieur reviennent dans la république [...] Ce n'est pas parce que nous sommes faibles (D5, 20.09.02)

Les propos pivotent autour des verbes "avaient", "étaient", "rentrent", "reviennent", "sommes" ayant pour sujets "la Côte d'lvoire", "ceux" et "nous". Tous expriment des états ${ }^{5}$ et des manières d'être de ces sujets. Dès les premières lignes de l'extrait, on constate qu'il y a une volonté de changement d'état invivable vers une position de bonne situation "sortir la tête de l'eau" qui est freinée par l'action d'élément perturbateur "terroriste" qui tend à empirer ou faire revenir à ladite situation invivable. L'association de verbe d'état et d'action dans le discours est une stratégie discursive qui vise à montrer l'adversaire sous un mauvais prisme. Le mot-verbe "avoir" reste exprimer des émotions et sentiments propres tandis que "être" dans le dernier énoncé joue le rôle de verbe-pont entre le sujet "nous" et son attribut "faibles". À la lecture de cet extrait, il appert que l'usage des verbes stratifie l'univers social contenu dans le discours et renforce les positionnements identitaires.

\subsubsection{Les verbes statifs et l'identité du partisan}

Les verbes statifs partisans sont tous les verbes lexicalement employés pour désigner l'univers de l'énonciateur et du peuple acquis à son idéologie politique. Ils sont surtout des éléments subjectifs. Toutes les occurrences relevées sont quasiment allouées à l'univers de l'énonciateur-peuple et très peu à l'adversaire socio-politique et les actants non-peuple. Analysons les extraits suivants:

Je ressens en ce moment comme une renaissance pour la Côte d'Ivoire [...] j'accepte de tenir la fonction du président de la République [...] je me rendrai dans le bureau présidentiel [...] (D2, 26.10.00) / Nous sommes réunis ce jour, chers compatriotes [...] Souvenons-nous des morts de Bouaflé, d'Agboville, de Treichville (D3, 09.11.00) / Honneurs à vous parce que vous êtes des héros. Mais la lutte n'est pas terminée. Restez mobilisés! (D6, ..10.02)

Les situations exprimées, ici, sont celles des sujets je, vous et nous référant, bien entendu, à l'énonciateur et au partisan. Les verbes "ressentir", "tenir", "rendre", "être", "souvenir" et "rester" rendant compte de leurs états ne sont, en fait, que des moyens détournés pour construire l'image de l'énonciateur. De l'expression des états émotionnels personnels et singuliers "je ressens", "j'accepte de tenir", il étend ses émotions propres à l'ensemble du corps-peuple en se confondant à lui "nous sommes réunis" et le rejoignant dans un devoir de mémoire "souvenons-nous". L'usage de nous,

\footnotetext{
5 Nous signalons que tous ces verbes, en gras, ne sont pas des verbes d'état. Dans la perspective connotative que nous avons de certains d'entre eux (rentrer, revenir et les tous autres) nous considérons qu'ils expriment un changement contextuel et situationnel d'un état A vers un état $B$. C'est justement pour cela que nous les appelons verbes-statifs-vicaires.
} 
n'est qu'une stratégie de légitimation de ses états et émotions subjectives. Dans cette dynamique, les verbes statifs jouent le rôle de ponts entre les individus mis en scène et l'univers de représentation: "vous êtes des héros" où ce dernier mot est attribut de vous et entre les deux le mot êtes (le verbe être) exprime non seulement l'état mais aussi la qualité. Le discours est alors affublé de mots presque laudatifs: "honneur à vous", visant à galvaniser l'auditoire en question. Ici, il s'agit des militaires: "restez mobilisés?'.

Tous les discours sont énoncés avec un contexte, une situation de communication qui déploie ses effets, ses émotions et ses attentes. L'énonciateur, membre de la communauté de destinataires des discours, prend en charge toute la scène énonciative et se construit une image qui tend à prendre en compte, de façon implicite, la communauté, le peuple Ivoirien. Les états contextuels et situationnels exprimés se cachent dans les 40 indices verbaux (statifs et statifs-vicaires) qui dégagent essentiellement une capacité à rendre sans effet les actions dirigées contre lui et son peuple. L'éthos de résistance et d'espérance, état et propriété, sont les images identitaires qui se logent au fond de cette distribution verbale que présente la Figure 4:

Figure 4 - Univers lexico-référentiel des verbes statifs partisans

\begin{tabular}{|c|}
\hline \\
Etat: résistance
\end{tabular}

Cet univers lexical présente dans un regard simpliste les états et autres propriétés dont l'énonciateur-peuple est sujet. De visu, les troubles engendrés par la crise semblent être euphémisés par le discours adressé à l'auditeur-peuple. Le populisme, en effet, "caractérisé par le sensationnel" (KAKDEU, 2013, p. 148), sait s'arranger et se présenter à son auditoire. Par-là, l'énonciateur joue sur la fibre patriotique de l'auditoire à qui il professe des lignes de vertus comme ici:

La valeur d'un grand pays c'est sa capacité à surmonter les affrontements [...] que nous trouvions en nous même, aujourd'hui, mais aussi demain, les capacités à surmonter les difficultés actuelles et les difficultés à venir $(\underline{D 4}, . .11 .01)$ 
Le verbe "surmonter", de son étymologie surmunter (vaincre, dépasser), à lui seul, fait un gros travail d'exaltation dans ce passage où il signifie nouvellement "ignorer, fairefi des difficultés pour avancer". L'appel à la résistance se dévoile subtilement à la deuxième occurrence du verbe et dans la fin des propos "les difficultés à venir". La simplicité énonciative par laquelle l'énonciateur exprime ces états fait dire à S. Mennell (2007, p. 113) qu'il prive l'auditeur-peuple de "la possibilité d'examiner les évènements subtils avec des grandes conséquences". Les évènements sont certes, présentés simplement mais, au fond, ce sont de gros bouleversements socio-politiques et militaires qui se cachent derrière ces verbes:

Nous ne sommes pas en face d'une mutinerie, nous sommes bien en face d'une tentative de coup d'Etat! (ㄷ, 20.09.02)

Le vocable "sommes", verbe être conjugué à la quatrième personne dans la perspective d'A. Niklas-Salminen (2012, p. 33), stipule l'état dans lequel se trouve l'énonciateur. Mais, à y voir de près, ces deux occurrences expriment des changements d'états, à savoir un état accompli "ne sommes pas" et un état actuel "sommes" qui continue à travers certains éléments syntaxiques. F. Martin (2006, p. 298) parle de verbes d'"accomplissements, qui d'écrivent un changement d'état prenant une certaine durée, et les achèvements, qui décrivent un évènement ponctuel". La première occurrence exprime un état accompli, par l'emploi concomitant de l'adverbe de négation "ne... pas" et du mot "sommes", l'attention du partisan est attiré sur le dépassement, l'audelà de la situation vécue; et lance, dans le même temps, l'état actuel "sommes bien en face" renforcé par le double usage adverbial de "bien" et de "en face" exprimant une gravité. Laquelle gravité simplement énoncée se trouve dans l'évolution crescendo des évènements décrits: de "mutinerie" à "coup d'état".

Au détour d'une proximité, tout verbe statif employé avec des outils de négation décrit un état très court: état accompli. En revanche, il présuppose dans son voisinage direct un autre changement d'état, dont il forme la frontière initiale. L'évènement frontière "mutinerie" est le denotatum de l'état grave "coup-d'état" dit état actuel. Les verbes statifs conférant ainsi au discours leur statut: on parle alors de discours statif. Ce discours est aussi valable dans la représentation de l'adversaire.

\subsubsection{Verbes statifs et l'identité adversative}

Les verbes statifs adversatifs sont ceux renvoyant à l'état, au caractère, à l'identité et la propriété de l'adversaire socio-politique de l'énonciateur. Très peu dans le corpus, les statifs adversatifs sont les ponts discursifs qui permettent à l'énonciateur d'atteindre ses adverses. Ils sont utilisés, avant tout, pour faire le procès de la place sociopolitique de l'adversaire. Les extraits ci-après en donnent la matérialité discursive: 
En posant un tel acte, il veut se mettre dans la voie de Baré Maïnassara. Ce n'est pas la voie que nous avons choisie $(\underline{D 1}, 24.10 .00)$ / Le problème Ouattara devenait empoisonnant pour tout le monde [...] Quand on n'est pas courageux, on ne fait pas la politique $(\underline{\mathrm{D} 4, . .11 .01)}$ / La Côte d'Ivoire a été trahie par ceux qui ont eu la liberté de parole et qui ont préféré faire parler les armes (D8, 30.12.02)

"Posant", "est", "devenait", "préféré" sont les indicateurs des états et propriétés de l'adversaire. Le mode de désignation de cette classe adverse reste le même: l'innommation. A priori, le verbe "être" vise plus à ressortir une nature quelque peu spécifique de l'adversaire: déroutant et peureux; comme le montrent ces énoncés: "ce n'est pas la voix que nous avons choisi", "on n'est pas courageux".

Ils expriment des états accomplis. Pour le premier, le statif est utilisé pour indiquer un éloignement, un écartement à la conduite communautaire (communauté représentée par nous) qui n'est, en vérité, que la résultante de l'accomplissement d'une action "en posant cet acte". Le verbe "poser" n'entre dans toute sa valeur sémique de verbe statifvicaire qu'en servant d'outil référentiel à l'acte posé par le nommé "Baré Mainassara".

L'utilisation de verbes d'actions comme verbes d'états contribue à donner à l'adversaire une image dévalorisante. Par ce procédé, en effet, l'énonciateur assimile la nature, le caractère, l'être de celui-ci à ses actions. La deuxième occurrence assure une fonction de pont entre le nommeur "on" et son attribut "courageux". Malheureusement les outils de négation viennent nier et transformer cette qualité en une identité infectée. C. Kerbrat-Orecchioni (1984, p. 213) parle de "discours apologétique et polémique, dont l'enjeu est de dévaloriser la position discursive de l'adversaire tout en valorisant la sienne".

Derrière ce projet que cachent ces ponts discursifs se trouve, en effet, l'affirmation d'un éthos de "respectueux des lois communautaires" et de "courageux". Pour tous les verbes statifs adversatifs le projet demeure le même: ressortir les changements d'état et de propriété dont l'adversaire peut être sujet. Ils fonctionnent dans un ensemble commutatif. Tous les états exprimés, a priori, se valent. Cependant, selon le projet qui est de démolir l'identité de l'adversaire, c'est par les attributs et autres compléments liés aux verbes statifs dans le champ discursif que la différence sémantique s'opère. Du coup, tous les verbes statifs adversatifs sont les contraires des verbes statifs partisans. Le combat de positionnement et de représentation de l'univers socio-politique se déroule dans ce que C. Kerbrat-Orecchioni (1984) appelle le "discours du parti, donc de parti pris".

\subsubsection{Verbes statifs-extérieur: de l'identité mitigée}

Pour rappel, un verbe statif ou attributif est un type sémantique de verbe dont le procès indique que son sujet possède une propriété particulière tel que l'état, le devenir, 
la façon d'être, etc. Ce sont des formes verbales qui n'expriment aucune action (en général) et ne fournissent qu'une description d'une condition. Les verbes statifs extérieurs sont tous les verbes qui retranscrivent les états, les qualités et les propriétés du groupe extérieur à la population directe de la crise ivoirienne. Un verbe ne rentre dans une catégorie actancielle que par considération des accessoires énonciatifs qui l'entourent. Analysons l'extrait suivant:

John Kufuor qui est aujourd'hui président en exercice de la CEDEAO (D9, ..02.03) / Le GTI est incapable de nous aider à désarmer les rebelles $(\underline{D 15}, . .09 .06)$ / Blaise Compaoré est d'accord, Toumani Touré est d'accord (D17, 30.07.07)

Le principal verbe dans ces énoncés c'est le verbe être conjugué, ici, à la troisième personne. Les quatre occurrences en présence exprime chacune une nature différente du fait des sujets: "John Kufuor", "Le GTr", "Blaise Compaoré", "Toumani Touré", qui revêtent eux-mêmes des caractères et des spécificités divers et différents. Ainsi le verbe "être" participe à situer les qualités et propriétés de chaque sujet qui le précède.

Dès la deuxième occurrence, on peut voir qu'il est révélateur d'une tare dévoilée dans l'adjectif "incapable". II joue le rôle de pont entre le sujet et cet adjectif. Les autres occurrences révèlent des qualités-fonctions "président en exercice de la CEDEAO" et des consensus d'accompagnement: "d'accord", dans la voie empruntée par l'énonciateur. Toutes les occurrences sont des états actuels et actualisés du fait de leur durée ou du moins, du temps de conjugaison utilisé pour les énoncer: le présent de l'indicatif. De par ces verbes et selon le corpus, ces actants sont restés dans une logique de recherche de solution à la crise ivoirienne à l'exception d'une frange pour qui l'énonciateur éprouve une certaine apathie.

Le discours statif devient le lieu de croisement et de confrontation de tous ces caractères. Toutes les énonciations cachent une subjectivité qui poursuit le projet de valorisation de son image dans toute sa dimension. Le choix d'accessoires énonciatifs n'est pas ex-nihilo car c'est le lieu de positionner le non-je et renforcer la valeur de vérité des propos tenus. Dans cette perspective, chaque déclaration s'évertue à donner des preuves, des détails à l'effet d'agir sur l'auditoire. II serait une véritable gageure de s'épancher sur toutes les déclarations de Laurent Gbagbo mais il n'est pas impossible d'étudier quelques verbes déclaratifs pour renforcer nos analyses sur les verbes statifs.

\subsection{Verbes déclaratifs}

Est dit déclaratif un "verbe qui énonce une simple communication (dire, expliquer, etc)"6. L'ensemble des items verbaux qui assertent sur une action, un état, un être, un

\footnotetext{
${ }^{6}$ Consulté le 12 avril 2016 sur http://www.cnrtl.fr/definition/verbedeclaratif
} 
évènement dans le corpus est dit verbe déclaratif. II y en a pour 3045 occurrences soit $21,5 \%$ de la surface verbal du corpus.

Soit l'extrait suivant:

Je marche, je marche, et je marcherai toujours (D2, 26.10.00) / nous devons prendre l'engagement solennel de ne céder ni à la rancœur, ni à la rancune (D3. $\underline{09.11 .00}$ ) / Nous disons non! [...] je leur dis, l'heure du patriotisme a sonné! (모, 20.09.02) / De toutes les crises, nous devons être capables d'en sortir [...] nous puissions mettre en place notre administration dans les régions de Bouaké et de Korhogo $(\underline{D 6}, . .10 .02)$

Les actes déclaratifs tournent autour de: "marche, marcherai", "devons", "disons, dis", "puissions" ayant pour sujets "je" et "nous". Le premier opérateur est un verbe d'action. "Marcher" est un verbe qui permet d'exprimer un mouvement, celui d'aller à pieds. Mais, dès que l'énonciateur l'utilise le verbe change de catégorie lexicale et passe de verbe d'action à verbe déclaratif: on parle d'opérateur déclaratif ambigu. Et il signifie dans ce contexte: “lutter, combattre par les idées, par les revendications". L'information contenue dans cette ambiguïté traduit un éthos de persistance et de persévérance.

Dans l'exemple suivant:

Houphouët m'a dit: "hélas! Tu me ressembles". II me parlait de toutes ses tournées à l'intérieur [...] On dit aussi que l'article 35 de la constitution est fait pour éliminer Ouattara [...] Ouattara lui, il dit: "Gbagbo n'a pas tenu ses promesses" (ㅁ, ...11.01)

Les opérateurs "dit" et "parlait" ont pour sujets "Houphouët", "il", "on", "Ouattara". L'indicateur Houphouët participe plus à renforcer l'image de l'énonciateur qu'à la mise en scène d'une opposition. Ensuite, le "on" qui réfère, ici, à un adversaire, montre une sorte de dédain de la part de l'énonciateur vis-à-vis de toutes les déclarations antérieures de cet adversaire-monde innommé. L'emphase énonciative dans "Ouattara lui, il" vise à ressortir une spécificité identitaire de l'individu. G. M. Irié Bi (2016 p. 165) d'"une affectation pompeuse, exagérée, accentuée dans le discours, dans le débit, dans les gestes, dans les termes employés". Par ce style l'énonciateur poursuit le projet de disqualifier la personne et ses propos. C'est ce type d'emphase que G. M. Irié Bi (2016, p. 158) appelle "emphase par dislocation". Dislocation de l'identité pour disloquer tout un univers référentiel.

\section{Conclusion}

La dynamique verbale est un vaste champ de valeurs que l'étude a ressorti dans les catégories référentielles qui sont en vérité des identités déguisées. Laurent Gbagbo, dans sa communication met constamment son auditoire à contribution par maints réglages linguistiques. Les verbes donnent au discours des colorations diverses par 
leurs valeurs connotatives. Les discours sont ainsi parsemés de stéréotypes et de positions changeants selon le contexte et l'auditoire en face. La forte prédominance des factifs (44\%) montre que le locuteur est un homme d'actions qui ne cesse de communiquer sur ses états et l'état de son pays à travers les statifs (33\%) dans des déclarations (22\%) omniprésentes dans le quotidien sociopolitique ivoirien. L'étude de ces classes verbales a permis de ressortir des éthos à portée politique, sociale, résistante, répulsive et restauratrice. Les motions de subjectivité qui se déploient dans ces classes verbales à coloration identitaire convergent toutes vers la valorisation de l'image-identité de Laurent Gbagbo vis-à-vis des autres actants dans l'espace de la crise ivoirienne

\section{Références}

CHARAUDEAU Patrick. Le discours politique: les masques du pouvoir. Paris: Vuibert, 2005.

CHARAUDEAU Patrick; MAINGUENEAU Dominique. Dictionnaire d'analyse du discours. Paris: éditions du Seuil, 2002.

DESMARIS Céline; MOSCAROLA Jean. Analyse de contenu et analyse lexicale: le cas d'une étude en management public. Lexicometrica, n.esp. Actes du colloque L'analyse de données textuelles: De l'enquête aux corpus littéraires, 2004. Disponible sur: http://lexicometrica.univ-paris3.fr/thema/thema7/Texte-Moscarola.pdf. Consulté: le 22 mars. 2015

DORNA Alexandre. Les effets langagiers du discours politique. Hermes, n. 16, 1995.

DUBOIS Jean, GIACOMO Mathée, GUESPIN Louis, MARCELLESI Christiane, MARCELLESI Jean-Baptiste et MEVEL Jean-Pierre. Le Dictionnaire de Linguistique et des sciences du langage. Paris: Larousse, 2018.

DUBOIS, Jean; LAGANE, René. Grammaire, toutes les notions fondamentales, toutes les règles clairement expliquées, analyse grammaticale et logique. Paris: Larousse, 2005.

FABIENNE, Martin. Prédicats statifs, causatifs et résultatifs en discours. Sémantique des adjectifs évaluatifs et des verbes psychologiques. Thèse (Doctorat en Linguistique) Université libre de Bruxelles, 2006. Disponible sur: https://tel.archives-ouvertes.fr/tel00450803. Consultée: le 21 juin. 2016.

GAVARD-PERRET Marie-Laure; MOSCAROLA Jean. Enoncé et ou énonciation? deux objets différents dans l'analyse lexicale en marketing. SAGE Publications, n. 13, v. 2, p.3147, 1998. DOI: https://doi.org/10.1177/076737019801300203

GHIGLIONE, Rodolphe; BROMBERG, Marcel; FRIEMEL, Edouard; KEKENBOSCH, Christiane; VERSTIGGEL, Jean-Claude. Prédications d'état, de déclaration et d'action: essai de classification en vue d'une application en analyse de contenu. Langages, 25e année, $n$. 100 , p. $81-100, \quad 1990$. Disponible sur: http://www.persee.fr/doc/lgge_0458726x_1990_num_25_100_1568. Consulté: le 31 mai 2016. 
GHIGLIONE, Rodolphe; MATALON, Benjamin; BACRI, Nicole. Les dires analysés: l'analyse propositionnelle du discours. Paris: PUV, 1985.

IRIÉ BI, Gohy Mathias. Alchimie de l'inversion dans la littérature orale: pour une économie linguistique du genre poétique Didiga. Abidjan, CERAP, 2016.

KAKDEU, Louis-Marie. L'expression de la violence dans le discours politique ivoirien de 2002 à 2013, Argotica, 2013.

KERBRAT-ORECCHIONI, Catherine. Discours politique et manipulation: du bon usage des contenus implicites. Paris: Armand Colin, 1984.

KOFFI-LÉZOU, Aimée-Danielle. Réalisations syntaxiques et discursives de l'ethos dans le discours politique. Baobab - Revue des sciences de l'imaginaire, arts, lettres et sciences humaines, 2012. Disponible sur: http://www.revuebaobab.org/content/view/207/33/

MENNELL, Stephens. L'Histoire des nouvelles. New York: Oxford University Press, 2007.

STASILO, Miroslav. Genre de discours politique en France et en Lituanie, éthos des politiciens: Déclarations et interviews des candidats élus et des candidats vaincus consécutives aux résultats des élections présidentielles (1993 - 2009). Thèse (Doctorat en Linguistique) - Université Paris-Est, 2012. Disponible sur https://tel.archivesouvertes.fr/tel00880255 Consultée le 25 août 2016

MONNERET, Philippe; RIOUL, René. Questions de syntaxe française. Paris: PUF, 2009.

NIKLAS-SALMINEN, Aïno. Le verbe. Paris: Armand Colin, 2012.

RIEGEL, Martin; PELLAT, Jean-Claude; RIOUL, René. Grammaire méthodique du français. Paris: PUF, 2009.

SINCLAIR, John. Corpus, concordance, collocation: Describing English language. Oxford: Oxford University Press, 1991. 
EID及A

Revista Eletrônica de Estudos Integrados em Discurso e Argumentação, n.21, v.3, 2021

Annexe 1 - Scenario 1: découpage du corpus

\begin{tabular}{|c|c|c|}
\hline Verbes: & Modalisations: & Pronoms: \\
\hline Factif $\quad 44.1 \%(6364)$ & Temps $18.9 \%(1076)$ & $34.4 \%(2628)$ \\
\hline Statif $\quad 33.2 \%(4787)$ & Lieu $\quad 13.9 \%(793)$ & $1.9 \% \quad(144)$ \\
\hline Déclaratif 21.5\% (3096) & Manière $6.9 \%$ (394) & $15.4 \%$ (1179) \\
\hline Performatif $1.2 \%$ (179) & Affirmation $4.3 \%$ (243) & “Nous" $17.2 \%$ (1314) \\
\hline & Doute $\quad 0.3 \% \quad(15)$ & "Vous" $8.0 \%$ (607) \\
\hline Connecteurs: & Négation $24.6 \%(1404)$ & “lls" $7.4 \% \quad(565)$ \\
\hline Condition 3.5\% (142) & Intensité 31.1\% (1775) & $9.2 \% \quad(703)$ \\
\hline Cause $\quad 15.5 \%(625)$ & & \\
\hline $3.6 \% \quad(145)$ & & \\
\hline Addition $43.0 \%$ (1730) & & \\
\hline Disjonction $3.4 \%$ (136) & Adjectifs: & \\
\hline Opposition $16.4 \%(658)$ & Objectif $39.8 \%$ (1444) & \\
\hline Comparaison $5.2 \%$ (211) & Subjectif $37.6 \%$ (1367) & \\
\hline Temps $9.4 \% \quad(377)$ & Numérique 22.6\% (821) & \\
\hline $0.0 \%(0)$ & & \\
\hline
\end{tabular}

Annexe 2 - Scenario 2: Comptage des verbes et leurs occurrences

\begin{tabular}{|c|c|c|c|c|c|c|c|}
\hline Etre & 2660 & Rendre & 0062 & Discuter & 0033 & Concerner & 0024 \\
\hline Avoir & 1046 & Passer & 0062 & Régler & 0033 & Citer & 0024 \\
\hline Faire & 0633 & Comprendre & 0057 & Poser & 0033 & Proposer & 0024 \\
\hline Dire & 0561 & Continuer & 0057 & Construire & 0033 & Retrouver & 0023 \\
\hline Aller & 0506 & Tenir & 0056 & Signer & 0033 & Ecouter & 0023 \\
\hline Vouloir & 0327 & Rester & 0053 & Attendre & 0033 & Adresser & 0023 \\
\hline Pouvoir & 0319 & Saluer & 0050 & Agir & 0033 & Respecter & 0023 \\
\hline Falloir & 0282 & Devenir & 0049 & Recevoir & 0032 & Envoyer & 0023 \\
\hline Venir & 0211 & Battre & 0049 & Oublier & 0031 & Gouverner & 0022 \\
\hline Prendre & 0151 & Partir & 0048 & Répondre & 0031 & Présenter & 0022 \\
\hline Voir & 0141 & Attaquer & 0048 & Elire & 0030 & Reprendre & 0022 \\
\hline Donner & 0136 & Aider & 0048 & Montrer & 0030 & Choisir & 0021 \\
\hline Demander & 0134 & Gagner & 0046 & Revenir & 0030 & Porter & 0021 \\
\hline Devoir & 0133 & Finir & 0044 & Former & 0029 & Ecrire & 0020 \\
\hline Savoir & 0125 & Payer & 0043 & Entrer & 0029 & Vendre & 0020 \\
\hline Mettre & 0116 & Accepter & 0043 & Soutenir & 0029 & Arrêter & 0020 \\
\hline Parler & 0116 & Organiser & 0043 & Tomber & 0026 & Echouer & 0020 \\
\hline Trouver & 0082 & Tuer & 0042 & Tirer & 0026 & Juger & 0019 \\
\hline Connaître & 0081 & Regarder & 0041 & Engager & 0026 & Réussir & 0019 \\
\hline Appeler & 0076 & Permettre & 0038 & Changer & 0026 & Lancer & 0018 \\
\hline Sortir & 0074 & Créer & 0037 & Mourir & 0026 & Rappeler & 0018 \\
\hline Chercher & 0069 & Vivre & 0037 & Perdre & 0026 & Réclamer & 0018 \\
\hline Travailler & 0069 & Bénir & 0036 & Rencontrer & 0026 & Armer & 0017 \\
\hline Remercier & 0067 & Compter & 0036 & Salir & 0026 & Terminer & 0017 \\
\hline Laisser & 0067 & Inviter & 0035 & Réunir & 0025 & Négocier & 0017 \\
\hline Arriver & 0065 & Voter & 0035 & Marcher & 0025 & Fonctionner & 0016 \\
\hline Commencer & 0064 & Souhaiter & 0034 & Essayer & 0025 & Désarmer & 0016 \\
\hline Penser & 0064 & Entendre & 0034 & Nommer & 0025 & Produire & 0016 \\
\hline Croire & 062 & Décider & 0034 & Inscrire & 0025 & Jouer & 0016 \\
\hline
\end{tabular}




\begin{tabular}{|c|c|c|c|c|c|c|c|}
\hline Constituer & 0016 & Accompagner & 0011 & Circuler & 0008 & Disposer & 0006 \\
\hline Occuper & 0016 & Déposer & 0011 & Rechercher & 0008 & Accorder & 0006 \\
\hline Tromper & 0016 & Lever & 0011 & Commettre & 0008 & Promener & 0006 \\
\hline Exister & 0016 & Adopter & 0011 & Retourner & 0008 & Envisager & 0006 \\
\hline Amener & 0015 & Servir & 0011 & Considérer & 0008 & Rédiger & 0006 \\
\hline Combattre & 0015 & Constater & 0011 & Veiller & 0007 & Choquer & 0006 \\
\hline Appliquer & 0015 & Répéter & 0010 & Manquer & 0007 & Estimer & 0006 \\
\hline Rentrer & 0015 & Abandonner & 0010 & Léguer & 0007 & Mentir & 0006 \\
\hline Achever & 0015 & Corriger & 0010 & Défendre & 0007 & Raconter & 0006 \\
\hline Préparer & 0015 & Durer & 0010 & Déclarer & 0007 & Modifier & 0006 \\
\hline Annoncer & 0014 & Indiquer & 0010 & Lier & 0007 & Monter & 0006 \\
\hline Apporter & 0014 & Dérouler & 0010 & Accuser & 0007 & Multiplier & 0006 \\
\hline Exprimer & 0014 & Partager & 0010 & Affirmer & 0007 & Mesurer & 0006 \\
\hline Transformer & 0014 & Manger & 0010 & Héler & 0007 & Quitter & 0006 \\
\hline Proclamer & 0014 & Utiliser & 0010 & Déplacer & 0007 & Entamer & 0006 \\
\hline Maintenir & 0014 & Avancer & 0010 & Dépasser & 0007 & Réfléchir & 0006 \\
\hline Descendre & 0014 & Assurer & 0010 & Sentir & 0007 & Observer & 0006 \\
\hline Lutter & 0014 & Appartenir & 0010 & Soulever & 0007 & Jeter & 0006 \\
\hline Apprendre & 0014 & Courir & 0010 & Soumettre & 0007 & Rejeter & 0006 \\
\hline Mener & 0014 & Rebeller & 0010 & Tracer & 0007 & Réhabiliter & 0006 \\
\hline Obliger & 0013 & Dormir & 0009 & Causer & 0007 & Limiter & 0006 \\
\hline Bâtir & 0013 & Étudier & 0009 & Refuser & 0007 & Aborder & 0005 \\
\hline Souffrir & 0013 & Acheter & 0009 & Informer & 0007 & Evoquer & 0005 \\
\hline Représenter & 0013 & Mériter & 0009 & Intervenir & 0007 & Suspendre & 0005 \\
\hline Souvenir & 0013 & Saisir & 0009 & Investir & 0007 & Assassiner & 0005 \\
\hline Aimer & 0013 & Asseoir & 0009 & Effectuer & 0007 & Financer & 0005 \\
\hline Développer & 0013 & Sembler & 0009 & Consolider & 0007 & Rire & 0005 \\
\hline Fier & 0013 & Déterminer & 0009 & Critiquer & 0007 & Etablir & 0005 \\
\hline Frapper & 0013 & Libérer & 0009 & Parcourir & 0007 & Voler & 0005 \\
\hline Soigner & 0013 & Demeurer & 0009 & Viser & 0007 & Fixer & 0005 \\
\hline Trahir & 0012 & Désigner & 0009 & Poursuivre & 0007 & Entraîner & 0005 \\
\hline Reconnaître & 0012 & Bloquer & 0009 & Pousser & 0007 & Sauver & 0005 \\
\hline Découvrir & 0012 & Appuyer & 0009 & Profiter & 0007 & Accueillir & 0005 \\
\hline Placer & 0012 & Subir & 0009 & Emprunter & 0007 & Triompher & 0005 \\
\hline Prévoir & 0012 & Diriger & 0009 & Opposer & 0006 & Affecter & 0005 \\
\hline Ramener & 0012 & Prononcer & 0009 & Aboutir & 0006 & Etendre & 0005 \\
\hline Rassembler & 0012 & Installer & 0008 & Accéder & 0006 & Surveiller & 0005 \\
\hline Atteindre & 0012 & Encourager & 0008 & Téléphoner & 0006 & Faillir & 0005 \\
\hline Pendre & 0012 & Emmener & 0008 & Cacher & 0006 & Unir & 0005 \\
\hline Lire & 0012 & Offrir & 0008 & Tenter & 0006 & Visiter & 0005 \\
\hline Faire-en-sorte & 0012 & Interdire & 0008 & Aller-mal & 0006 & Arranger & 0005 \\
\hline Cesser & 0012 & Empêcher & 0008 & Encadrer & 0006 & Transférer & 0005 \\
\hline Suivre & 0012 & Issir & 0008 & Tendre & 0006 & Supprimer & 0005 \\
\hline Crier & 0011 & Éclater & 0008 & Pardonner & 0006 & Embêter & 0005 \\
\hline Protéger & 0011 & Mettre-fin & 0008 & Gérer & 0006 & Remettre & 0005 \\
\hline Conduire & 0011 & Ouvrir & 0008 & Consulter & 0006 & Initier & 0005 \\
\hline Suffire & 0011 & Dresser & 0008 & Succéder & 0006 & Cultiver & 0005 \\
\hline Mobiliser & 0011 & Importer & 0008 & Signaler & 0006 & Instaurer & 0005 \\
\hline Condamner & 0011 & Exercer & 0008 & Détruire & 0006 & Honorer & 0005 \\
\hline Expliquer & 0011 & Diviser & 0008 & Rétablir & 0006 & Programmer & 0005 \\
\hline Eviter & 0011 & Relever & 0008 & Résoudre & 0006 & Remplir & 0005 \\
\hline Féliciter & 0011 & Prêter & 0008 & Renforcer & 0006 & Imposer & 0005 \\
\hline Obtenir & 0011 & Brûler & 0008 & Survenir & 0006 & Relancer & 0005 \\
\hline Valoir & 0011 & Toucher & 0008 & Distinguer & 0006 & Réaliser & 0005 \\
\hline Contrer & 0011 & Traverser & 0008 & Renverser & 0006 & Refaire & 0005 \\
\hline Ressembler & 0011 & Conclure & 0008 & Reprocher & 0006 & Rectifier & 0005 \\
\hline Espérer & 0011 & Confier & 0008 & Guérir & 0006 & Livrer & 0005 \\
\hline
\end{tabular}




\begin{tabular}{|c|c|c|c|c|c|c|c|}
\hline Promettre & 0005 & Hériter & 0004 & Contenter & 0003 & Prétexter & 0003 \\
\hline Délivrer & 0005 & Exiger & 0004 & Dénoncer & 0003 & Ressaisir & 0003 \\
\hline Rassurer & 0005 & Intégrer & 0004 & Infliger & 0003 & Réparer & 0003 \\
\hline Laver & 0005 & Examiner & 0004 & Charger & 0003 & Couvrir & 0003 \\
\hline Résister & 0005 & Dégager & 0004 & Compromettre & 0003 & OEuvrer & 0003 \\
\hline Parvenir & 0005 & Assumer & 0004 & Témoigner & 0003 & Préciser & 0003 \\
\hline Réserver & 0005 & Ecarter & 0004 & Taire & 0003 & Consacrer & 0003 \\
\hline Garantir & 0005 & Démarrer & 0004 & Taper & 0003 & Secouer & 0003 \\
\hline Conserver & 0005 & Célébrer & 0004 & Ambitionner & 0003 & Semer & 0003 \\
\hline Garder & 0005 & Débattre & 0004 & Diminuer & 0003 & Justifier & 0003 \\
\hline Prier & 0005 & Blesser & 0004 & Fonder & 0003 & Conjurer & 0003 \\
\hline Bitumer & 0005 & Autoriser & 0004 & Comploter & 0003 & Elever & 0003 \\
\hline Conférer & 0005 & Décourager & 0004 & Remonter & 0003 & Accroître & 0003 \\
\hline Conter & 0005 & Consentir & 0004 & Gifler & 0003 & Accréditer & 0003 \\
\hline Coûter & 0005 & Communiquer & 0004 & Concourir & 0003 & Transposer & 0003 \\
\hline Recruter & 0004 & Apprêter & 0004 & Handicaper & 0003 & Trancher & 0003 \\
\hline Plaire & 0004 & Distraire & 0004 & Apparaître & 0003 & Traiter & 0003 \\
\hline Employer & 0004 & Dissoudre & 0004 & Redonner & 0003 & Evoluer & 0003 \\
\hline Endeuiller & 0004 & Amuser & 0004 & Fuir & 0003 & Eliminer & 0003 \\
\hline Projeter & 0004 & Analyser & 0004 & Surmonter & 0003 & Rapprocher & 0003 \\
\hline Enseigner & 0004 & Démontrer & 0004 & Noter & 0003 & Disparaître & 0003 \\
\hline Enregistrer & 0004 & Arracher & 0004 & $\operatorname{Re}$ & 0003 & Rapporter & 0003 \\
\hline Préférer & 0004 & Approuver & 0004 & Marier & 0003 & Excuser & 0003 \\
\hline Nécessiter & 0004 & Distribuer & 0004 & Boucler & 0003 & Entreprendre & 0003 \\
\hline Nuire & 0004 & Approprier & 0004 & Conseiller & 0003 & Recouvrer & 0003 \\
\hline Prolonger & 0004 & Accomplir & 0004 & Piétiner & 0003 & Vérifier & 0003 \\
\hline Marquer & 0004 & Collecter & 0004 & Mander & 0003 & Exposer & 0003 \\
\hline Graver & 0004 & Commander & 0004 & Pratiquer & 0003 & Verser & 0003 \\
\hline Fatiguer & 0004 & Embrasser & 0004 & ouler & 0003 & Enrichir & 0003 \\
\hline Substituer & 0004 & Emouvoir & 0004 & Méditer & 0003 & Transporter & 0003 \\
\hline Habiter & 0004 & Contourner & 0004 & Mettre-en-œuvr & 0003 & Exécuter & 0003 \\
\hline Renvoyer & 0004 & Percevoir & 0003 & Décéder & 0003 & Enlever & 0003 \\
\hline Générer & 0004 & Dépendre & 0003 & uiller & 0003 & Electrifier & 0003 \\
\hline Forcer & 0004 & Cadrer & 0003 & Rejoindre & 0003 & Efforcer & 0003 \\
\hline Retirer & 0004 & Incarner & 0003 & Augmenter & 0003 & Adapter & 0003 \\
\hline Retenir & 0004 & Participer & 0003 & Attirer & 0003 & Empoisonner & 0003 \\
\hline Gâter & 0004 & Déplorer & 0003 & Décentraliser & 0003 & En-vouloir & 0003 \\
\hline Résulter & 0004 & Confirmer & 0003 & Ressentir & 0003 & Acquérir & 0003 \\
\hline Réjouir & 0004 & Implanter & 0003 & Rompre & 0003 & Emparer & 0003 \\
\hline Tourner & 0004 & Incliner & 0003 & Prétendre & 0003 & Actionner & 0003 \\
\hline Légitimer & 0004 & Rembourser & 0003 & Oser & 0003 & Echanger & 0003 \\
\hline Reculer & 000 & Boucher & 0003 & Couronner & 0003 & Eloigner & 0003 \\
\hline Réduire & 0004 & Sommer & 0003 & Ordonner & 0003 & Eriger & 0 \\
\hline Remarquer & 0004 & Oter & 0003 & Solliciter & 0003 & & \\
\hline Fâcher & 0004 & Souligner & 0003 & Instruire & 0003 & Réconcilier & 0 \\
\hline
\end{tabular}

